(1)

CrossMark

\title{
Thrombosis and COVID-19 pneumonia: the clot thickens!
}

\author{
Laura C. Price ${ }^{1,2}$, Colm McCabe ${ }^{1,2}$, Ben Garfield ${ }^{1}$ and Stephen J. Wort ${ }^{1,2}$ \\ Affiliations: ${ }^{1}$ Royal Brompton and Harefield NHS Trust, London, UK. ${ }^{2}$ National Heart and Lung Institute, \\ Imperial College, London, UK.
}

Correspondence: Laura C. Price, Royal Brompton and Harefield NHS Trust, Sydney Street, London, SW3 6NP, UK. E-mail laura.pricedrbht.nhs.uk

@ERSpublications

Pulmonary thrombosis appears to be common in COVID-19 pneumonia and takes two forms, proximal pulmonary emboli and/or distal thrombosis. The possible mechanisms and clinical implications are discussed. https://bit.ly/372Xdhw

Cite this article as: Price LC, McCabe C, Garfield B, et al. Thrombosis and COVID-19 pneumonia: the clot thickens! Eur Respir J 2020; 56: 2001608 [https://doi.org/10.1183/13993003.01608-2020].

At the end of last year, a novel coronavirus, severe acute respiratory syndrome coronavirus 2 (SARS-CoV-2), resulted in an acute respiratory illness epidemic in Wuhan, China [1, 2]. The World Health Organization (WHO) termed this illness coronavirus disease 2019 (COVID-19). The coronavirus family have been shown to enter cells through binding angiotensin-converting enzyme 2 (ACE-2), found mainly on alveolar epithelium and endothelium. Activation of endothelial cells is thought to be the primary driver for the increasingly recognised complication of thrombosis. Viral inclusion bodies have been identified in endothelial cells in a variety of organs, from the lung to the gastrointestinal tract [3]. The immune dysregulation characteristic of severe COVID-19 infection may be initiated by "pyroptosis", a particularly pro-inflammatory form of apoptosis initially described in macrophages [4], with rapid viral replication leading to massive release of inflammatory mediators. One of the most consistent findings is that of a raised D-dimer level. Although many inflammatory processes can influence D-dimer levels, it almost certainly reflects, to some extent, intravascular thrombosis in patients with COVID-19 [5, 6]. In the early studies emerging from China, an elevated D-dimer level $\left(>1000 \mathrm{ng} \cdot \mathrm{mL}^{-1}\right)$ at admission was associated with increased risk of in-hospital death [7]. An elevated D-dimer continues to be one of the most consistent markers of poor outcome [8].

The true prevalence of thrombosis associated with COVID-19 infection is unknown, as most studies to date do not include systematic and comprehensive investigation protocols. It appears that it is also important to consider the stage of the disease course and where the patient is tested, e.g. ward versus intensive care unit (ICU). In addition, both of these factors will influence the amount of anticoagulation a patient may receive, which in turn may influence subsequent thrombosis. Furthermore, most studies refer to pulmonary embolism (PE), whereas there is persuasive evidence to suggest much of this may be "in situ" pulmonary thrombosis [9]. Two recent Dutch studies have reported cumulative incidences of thrombotic events between 48 and $49 \%$ respectively in their ICUs in patients with COVID-19 pneumonia $[10,11]$. Most thrombotic events are described as PE, for instance $87 \%$ in the paper by KLOK et al. [12], although it should also be noted that venous and arterial thrombosis is described [10]. In this edition of the European Respiratory Journal, BOMPARD et al. [13] refine this further by describing a 50\% cumulative 
incidence of PE, diagnosed by computed tomography-pulmonary angiogram (CTPA), in COVID-19 patients admitted to the ICU in two hospitals of the University of Paris. It is important to note that all patients received thromboprophylaxis, which is a common theme in most reports. In fact, in one study $56 \%$ of patients already on full anticoagulation were diagnosed with PE [14].

Several studies have compared rates of thrombosis in ICU and non-ICU populations [11, 13, 15]. For instance, Middeldorp et al. [11], report a cumulative incidence of any venous thromboembolism (VTE) event (symptomatic or asymptomatic) of $10 \%$ at 2 weeks in a non-ICU population versus $48 \%$ in the ICU, whereas BOMPARD et al. [13] report an 18\% cumulative incidence of PE in non-ICU patients versus $50 \%$ in ICU. This is further supported by the study of CRIEL et al. [16], also published in this edition of European Respiratory Journal, comparing deep venous thrombosis (DVT) rates between ICU patients (13\%) and ward patients $(4 \%)$. One obvious question is whether these rates of thrombosis are higher than we would expect? Two studies have used a retrospective "comparative" non-COVID-19 ICU population and report 3-6 times the expected rates of thrombosis in the COVID-19 populations [17, 18]. There also appeared to be a very high association with thrombosis in SARS, another coronavirus, and higher than H1N1 for instance $[19,20]$. However, the described rates are probably consistent with those reported in patients with severe sepsis or shock [21]. It may be that there are just many more very sick patients in this pandemic. However, it is also clear that we are likely to be underestimating the true prevalence.

Several other interesting observations have been described. Firstly, in the study of Lodigiani et al. [15], around 50\% of patients were diagnosed with thrombosis within $24 \mathrm{~h}$ of admission, supporting the hypothesis that many patients are probably presenting with PE. Secondly, KLOK et al. [12] describe a small population who happened to be on long-term anticoagulation at admission, who appeared to be protected from developing thrombosis, although, interestingly, not overall mortality. Thirdly, there is considerable anecdotal evidence that patients are dying after discharge and that this could be thrombosis related.

In the accompanying editorial, HUERTAS et al. [22] elegantly describe the early pathogenesis in COVID-19 pneumonia defined by a widespread endotheliilitis affecting multiple organ systems. As such, viral inclusion bodies are observed within endothelial cells accompanied by apoptosis, inflammatory cell infiltration and microvascular thrombosis [23]. At the same time, systemic inflammation is commonly observed, with elevated levels of C-reactive protein, fibrinogen and cytokines such as interleukin-6 [24]. Although the exact mechanisms of COVID-19-induced thrombosis have not been elucidated, at least some of the well-described mechanisms associated with infection/inflammation are likely to be relevant [25], as illustrated in figure 1. These include the increased production of tissue factor and amplification of the coagulation cascade, resulting in increased production of thrombin and, consequently, fibrin. Small studies describing thromboelastography in patients with COVID-19 suggest clot formation is extremely rapid and also resistant to breakdown [26]. This, in turn, is likely to relate to reduced fibrinolysis due to increased production of plasminogen activator inhibitor-1 (PAI-1), again as a result of inflammation. Deposition of components of the complement system, such as C5b-9, in damaged vessels of COVID-19 patients indicate that this may be another important pro-thrombotic mechanism as it has been observed in other conditions associated with micro-thrombosis, such as anti-phospholipid syndrome [27]. Furthermore, neutrophil extracellular traps (NETs) have also been observed in vessels in autopsy specimens of patients with COVID-19 [28]. These are associated with high circulating levels of cell-free DNA and histones, which in turn can activate pro-thrombotic pathways leading to increased thrombin production [29]. In addition to ACE-2-mediated SARS-CoV-2 viral entry, recent reports of affinity of the SARS-CoV-2 spike protein and CD147, a membrane glycoprotein and extracellular matrix metalloproteinase inducer expressed on a variety of haematopoietic cell lines, suggest another potentially novel mechanism of thrombosis and inflammation in the arterial and venous circulations [30]. Finally, the profound hypoxaemia that is often observed is a likely driver of vasoconstriction, inflammation and thrombosis. Hypoxaemia will result in activation of hypoxia-inducible factors, which in turn will activate cytokines, tissue factor and PAI-1 [31, 32].

Overall, the high rate of pulmonary thrombosis in COVID-19 conceivably lies in the confluence of three processes: firstly, the intense endothelial inflammation described above, leading to "in situ" thrombosis, including microvascular thrombosis; secondly, altered pulmonary blood flow in response to the parenchymal process, disturbing Virchow's triad within the lung; and thirdly, classical DVT to PE transition, which may, in fact, be the minor partner [10].

As the recognition of the thrombotic complications of COVID-19 increases, more guidelines are emerging on their prevention, investigation and management $[33,34]$. As already stated, we are likely to be missing thrombotic complications in many patients. Most guidance recognises the importance of keeping the balance between providing quick, pragmatic advice to worried physicians keen to do the best for their patients, with the recognition that evidence for full anticoagulation is of course lacking in this population. 


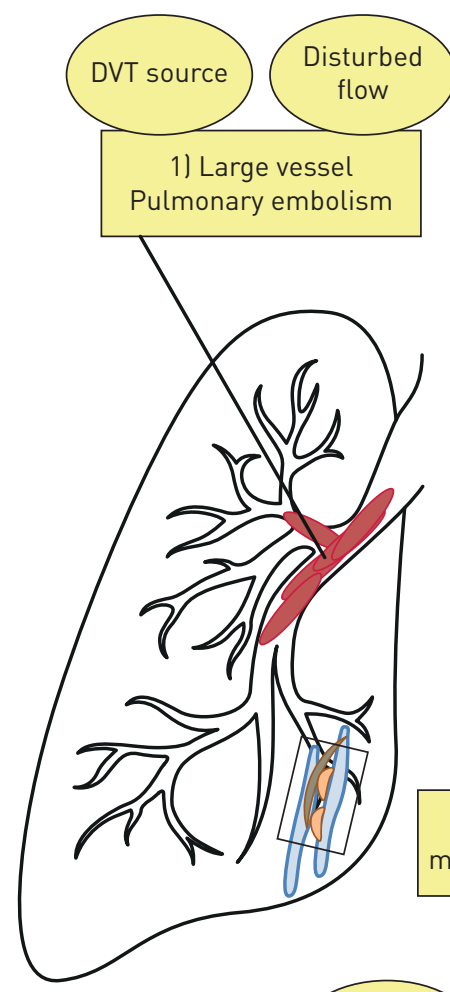

2) Distal pulmonary microvascular occlusion
Viral spike attachment to ACE-2 receptor

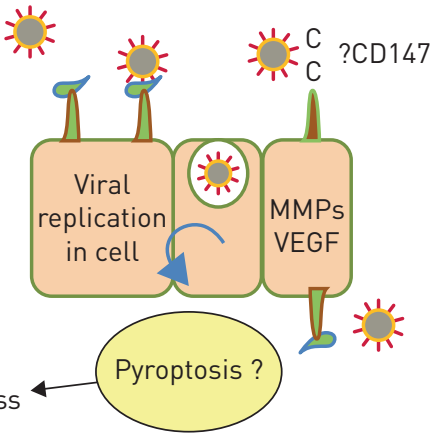

DAMPs Oxidative stress

Cytokine release from neighbouring epithelial cells, endothelial cells, alveolar macrophages
TF upregulated on platelets, monocytes, macrophages and EC in response to cytokines
Pro-inflammatory

feedback loop:

Cytokines and chemokines attact monocytes, macrophages, $T$ cells

Complement activation

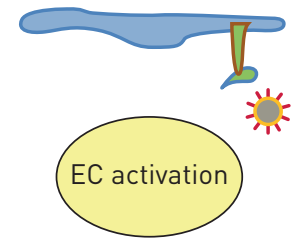

Inflammatory vascular injury induces thrombin formation
Downregulation of fibrinolytic pathways
Downregulation of anticoagulant pathways
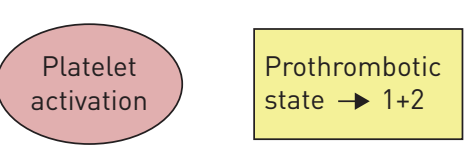

$\hookrightarrow$ Propagation of inflammation

PAI-1

(2) (2)

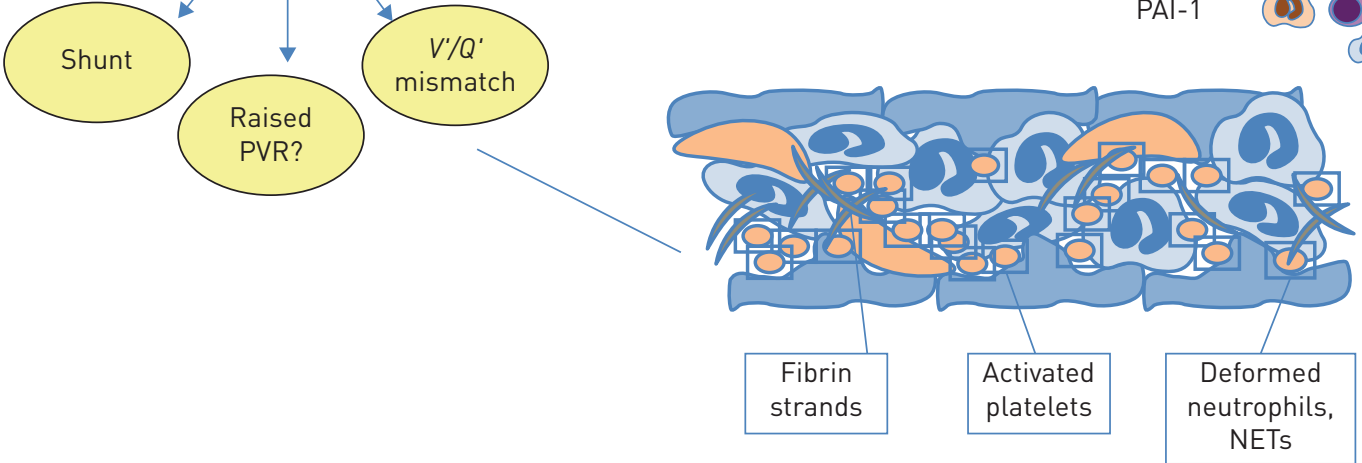

FIGURE 1 Hypothesis of the origin of coronavirus disease 2019 (COVID-19)-associated pulmonary emboli and lung microcirculatory thrombotic disease: interaction of inflammation and coagulation. When severe acute respiratory syndrome coronavirus 2 (SARS-CoV-2) infects cells expressing the surface receptors angiotensin-converting enzyme 2 (ACE-2), active replication and release of the virus may cause the host cell to undergo pyroptosis (pro-inflammatory apoptosis) and release damage-associated molecular patterns (DAMPs), activating oxidant stress, and generating pro-inflammatory cytokine and chemokine release from nearby epithelial cells, endothelial cells and alveolar macrophages. These proteins in turn attract inflammatory cells to the site of infection, promoting a pro-inflammatory feedback loop. Tissue factor, usually hidden on the subendothelium, is upregulated on platelets, leukocytes and endothelial cells (ECs) during inflammation, leading to activation of both the extrinsic and intrinsic coagulation pathways to make thrombin. Complement activation is also relevant. Thrombin binds to protease-activated receptors (e.g. PAR-1) to promote the formation of fibrin from fibrinogen, the activation of platelets and subsequent clot stabilisation, also propagating further inflammation. Natural anticoagulants and fibrinolytics may also be reduced in COVID-19/SARS-CoV-2 infection. Occluded small pulmonary blood vessels are likely to contain fibrin, platelets and coagulation factors, as well as neutrophils that become trapped in neutrophil extracellular traps (NETs) as they pass through the lung. Ongoing inflammation provides a positive feedback loop. Additional procoagulant stimuli include lung hypoxia, for example via upregulation of plasminogen activator inhibitor-1 (PAl-1) through suppression of fibrinolysis. In this prothrombotic pneumonitis or acute respiratory distress syndrome, whether similar mechanisms promote both microthrombosis as well as larger vessel pulmonary embolic disease is not known. 
Ideally, therefore, wherever practical, we believe the diagnosis of VTE in patients with COVID-19 should be made by traditional imaging modalities; CTPA and/or in the case of clinical suspicion of DVT, by compression venous Doppler of the lower limb [34]. There should be a high suspicion for thrombotic complications and low threshold for definitive imaging, where possible. Traditional D-dimer cut offs are unlikely to be useful in determining who should go on to have a CTPA due to an unacceptably low specificity which will be exacerbated by admission to ICU [5, 35]. However, for many reasons imaging may be impossible. D-dimer has been used in some institutions to guide anticoagulation, even in the absence of imaging detected VTE $[34,36]$. The risks and benefits of an approach aimed at targeting both imaging-detected VTE as well as "presumed thrombosis" is yet to be determined, but excess bleeding is an almost inevitable consequence [37]. Whether this excess risk is offset by improvements in overall survival is yet to be seen. Anticoagulation is clearly not the only treatment needed in patients with COVID-19 and in many patients, on the sicker end of the spectrum, may be too late to alter outcome. The intimate association between inflammation and thrombosis would suggest an anti-inflammatory/anti-viral therapeutic approach should be considered in parallel to anticoagulation. Furthermore, as the fibrinolytic potential of blood from patients with COVID-19 is extremely low, even with anticoagulation, immediate consequences of thrombus may not be treated. We may just be preventing further thrombus. Indeed, case reports describe the use of fibrinolytics in selected patients, with short-term benefits [38], and trials are on-going. But how long will this beneficial effect last in the procoagulant COVID-19 milieu? Histology from autopsy specimens demonstrate platelet aggregates obstructing the microvasculature. Thromboelastography and platelet function tests reveal that patients with COVID-19 have activated platelets [39], suggesting that there may be a role for anti-platelet therapy. Here, the potential to do benefit may be theoretically greater but so is the chance of doing harm.

Clearly results of ongoing trials are urgently needed to further inform clinical practice on the use of anticoagulation. This includes both patients admitted to ICUs and those at risk on the general ward. With regards to non-ICU patients, questions to be asked include: what are the triggers for more intense anticoagulation where imaging is not available? Can this be done using D-dimer alone and what is the threshold to use? What do you do with patients when they are discharged? In the meantime, local and national advice is available and being constantly updated [34]. Finally, with certainty, we can say that the more we know about COVID-19, the more the clot thickens!

Acknowledgement: The authors would like to acknowledge Deepa Arachchillage, consultant haematologist, Royal Brompton Hospital.

Conflict of interest: L.C. Price reports personal fees from Actelion and Johnson and Johnson, outside the submitted work. C. McCabe has nothing to disclose. B. Garfield has nothing to disclose. S.J. Wort reports grants and personal fees from Actelion Pharmaceuticals and Bayer, personal fees from MSD and GSK, outside the submitted work.

\section{References}

1 Huang C, Wang Y, Li X, et al. Clinical features of patients infected with 2019 novel coronavirus in Wuhan, China. Lancet 2020; 395: 497-506.

2 Chen N, Zhou M, Dong X, et al. Epidemiological and clinical characteristics of 99 cases of 2019 novel coronavirus pneumonia in Wuhan, China: a descriptive study. Lancet 2020; 395: 507-513.

3 Varga Z, Flammer AJ, Steiger P, et al. Endothelial cell infection and endotheliitis in COVID-19. Lancet 2020; 395: $1417-1418$.

4 Cookson BT, Brennan MA. Pro-inflammatory programmed cell death. Trends Microbiol 2001; 9: 113-114.

5 Leonard-Lorant I, Delabranche X, Severac F, et al. Acute pulmonary embolism in COVID-19 patients on CT angiography and relationship to D-dimer levels. Radiology 2020; in press [https://doi.org/10.1148/ radiol.2020201561].

6 Cui S, Chen S, Li X, et al. Prevalence of venous thromboembolism in patients with severe novel coronavirus pneumonia. J Thromb Haemost 2020; 18: 1421-1424.

7 Zhou F, Yu T, Du R, et al. Clinical course and risk factors for mortality of adult inpatients with COVID-19 in Wuhan, China: a retrospective cohort study. Lancet 2020; 395: 1054-1062.

8 Bikdeli B, Madhavan MV, Jimenez D, et al. COVID-19 and thrombotic or thromboembolic disease: implications for prevention, antithrombotic therapy, and follow-up. J Am Coll Cardiol 2020; 75: 2950-2973.

9 Wang T, Chen R, Liu C, et al. Attention should be paid to venous thromboembolism prophylaxis in the management of COVID-19. Lancet Haematol 2020; 7: e362-e363.

10 Klok FA, Kruip M, van der Meer NJM, et al. Incidence of thrombotic complications in critically ill ICU patients with COVID-19. Thromb Res 2020; 191: 145-147.

11 Middeldorp S, Coppens M, van Haaps TF, et al. Incidence of venous thromboembolism in hospitalized patients with COVID-19. J Thromb Haemost 2020; in press [https://doi.org/10.1111/jth.14888].

12 Klok FA, Kruip M, van der Meer NJM, et al. Confirmation of the high cumulative incidence of thrombotic complications in critically ill ICU patients with COVID-19: An updated analysis. Thromb Res 2020; 191: 148-150.

13 Bompard F, Monnier H, Saab I, et al. Pulmonary embolism in patients with COVID-19 pneumonia. Eur Respir J 2020; 56: 2001365

14 Llitjos JF, Leclerc M, Chochois C, et al. High incidence of venous thromboembolic events in anticoagulated severe COVID-19 patients. J Thromb Haemost 2020; 18: 1743-1746. 
15 Lodigiani C, Iapichino G, Carenzo L, et al., Venous and arterial thromboembolic complications in COVID-19 patients admitted to an academic hospital in Milan, Italy. Thromb Res 2020; 191: 9-14.

16 Criel M, Falter M, Jaeken J, et al. Venous thromboembolism in SARS-CoV-2 patients: only a problem in ventilated ICU patients, or is there more to it? Eur Respir J 2020; 56: 2001201.

17 Poissy J, Goutay J, Caplan M, et al. Pulmonary embolism in COVID-19 patients: awareness of an increased prevalence. Circulation 2020; 142: 184-186.

18 Helms J, Tacquard C, Severac F, et al. High risk of thrombosis in patients with severe SARS-CoV-2 infection: a multicenter prospective cohort study. Intensive Care Med 2020; 46: 1089-1098.

19 Lew TW, Kwek TK, Tai D, et al. Acute respiratory distress syndrome in critically ill patients with severe acute respiratory syndrome. JAMA 2003; 290: 374-380.

20 Chong PY, Chui P, Ling AE, et al. Analysis of deaths during the severe acute respiratory syndrome (SARS) epidemic in Singapore: challenges in determining a SARS diagnosis. Arch Pathol Lab Med 2004; 128: 195-204.

21 Kaplan D, Casper TC, Elliott CG, et al. VTE incidence and risk factors in patients with severe sepsis and septic shock. Chest 2015; 148: 1224-1230.

22 Huertas A, Montani D, Savale L, et al. Endothelial cell dysfunction: a major player in SARS-CoV-2 infection (COVID-19)? Eur Respir J 2020; 56: 2001634.

23 Varga Z, Flammer AJ, Steiger P, et al. Endothelial cell infection and endotheliitis in COVID-19. Lancet 2020; 395 $1417-1418$.

24 Chen G, Wu D, Guo W, et al. Clinical and immunological features of severe and moderate coronavirus disease 2019. J Clin Invest 2020; 130: 2620-2629.

25 Connors JM, Levy JH. COVID-19 and its implications for thrombosis and anticoagulation. Blood 2020; 135: 2033-2040.

26 Panigada M, Bottino N, Tagliabue P, et al. Hypercoagulability of COVID-19 patients in intensive care unit. A report of thromboelastography findings and other parameters of hemostasis. J Thromb Haemost 2020; 18: $1738-1742$

27 Magro C, Mulvey JJ, Berlin D, et al. Complement associated microvascular injury and thrombosis in the pathogenesis of severe COVID-19 infection: a report of five cases. Transl Res 2020; 220: 1-10.

28 Zuo Y, Yalavarthi S, Shi H, et al. Neutrophil extracellular traps in COVID-19. JCI Insight 2020; 5: 138999.

29 Barnes BJ, Adrover JM, Baxter-Stoltzfus A, et al. Targeting potential drivers of COVID-19: neutrophil extracellular traps. J Exp Med 2020; 217: e20200652.

30 Wang K, Chen W, Zhou Y-S, et al. SARS-CoV-2 invades host cells via a novel route: CD147-spike protein. BioRxiv 2020; preprint [https://doi.org/10.1101/2020.03.14.988345].

31 Yan SF, Mackman N, Kisiel W, et al. Hypoxia/hypoxemia-induced activation of the procoagulant pathways and the pathogenesis of ischemia-associated thrombosis. Arterioscler Thromb Vasc Biol 1999; 19: 2029-2035.

32 Gupta N, Zhao YY, Evans CE. The stimulation of thrombosis by hypoxia. Thromb Res 2019; 181: 77-83.

33 Thachil J, Tang N, Gando S, et al. ISTH interim guidance on recognition and management of coagulopathy in COVID-19. J Thromb Haemost 2020; 18: 1023-1026.

34 Condliffe R, Bunclark K, Hurdman J, et al. BTS Guidance on Venous Thromboembolic Disease in patients with COVID-19. British Thoracic Society, 2020. Available from: https://www.brit-thoracic.org.uk/about-us/ covid-19-information-for-the-respiratory-community/

35 Minet C, Potton L, Bonadona A, et al. Venous thromboembolism in the ICU: main characteristics, diagnosis and thromboprophylaxis. Crit Care 2015; 19: 287.

36 Cohoon KP, Mahé G, Tafur AJ, et al. Emergence of institutional antithrombotic protocols for coronavirus 2019. Res Pract Thromb Haemost 2020; 4: 510-517.

37 Klok FA, Kooiman J, Huisman MV, et al. Predicting anticoagulant-related bleeding in patients with venous thromboembolism: a clinically oriented review. Eur Respir J 2015; 45: 201-210.

38 Wang J, Hajizadeh N, Moore EE, et al. Tissue plasminogen activator (tPA) treatment for COVID-19 associated acute respiratory distress syndrome (ARDS): a case series. J Thromb Haemost 2020; 18: 1752-1755.

39 Song JC, Wang G, Zhang W, et al. Chinese expert consensus on diagnosis and treatment of coagulation dysfunction in COVID-19. Mil Med Res 2020; 7: 19. 